\title{
A Prospective Mixed-Methods Study of Decision-Making on Surgery or Active Surveillance for Low-Risk Papillary Thyroid Cancer
}

\author{
Anna M. Sawka, ${ }^{1}$ Sangeet Ghai, ${ }^{2}$ Tom Yoannidis, ${ }^{3}$ Lorne Rotstein, ${ }^{4}$ Patrick J. Gullane, ${ }^{5}$ Ralph W. Gilbert, \\ Jesse D. Pasternak, ${ }^{4}$ Dale H. Brown, ${ }^{5}$ Antoine Eskander, ${ }^{6}$ John R. de Almeida, ${ }^{5}$ Jonathan C. Irish, \\ Kevin Higgins, ${ }^{6}$ Danny J. Enepekides, ${ }^{6}$ Eric Monteiro, ${ }^{7}$ Avik Banerjee, ${ }^{8}$ Manish Shah, ${ }^{5,9}$ Everton Gooden, ${ }^{9}$ \\ Afshan Zahedi, ${ }^{10}$ Mark Korman, ${ }^{11}$ Shereen Ezzat, ${ }^{12}$ Jennifer M. Jones, ${ }^{13}$ Valeria E. Rac, ${ }^{14-17}$ \\ George Tomlinson, ${ }^{16,17}$ Aleksandra Stanimirovic, ${ }^{15,16}$ Amiram Gafni, ${ }^{18}$ \\ Nancy N. Baxter ${ }^{19}$ and David P. Goldstein ${ }^{5}$
}

Background: Active surveillance (AS) of small, low-risk papillary thyroid cancers (PTCs) is increasingly being considered. There is limited understanding of why individuals with low-risk PTC may choose AS over traditional surgical management.

Methods: We present a mixed-methods analysis of a prospective observational real-life decision-making study regarding the choice of thyroidectomy or AS for management of localized, low-risk PTCs $<2 \mathrm{~cm}$ in maximum diameter (NCT03271892). Patients were provided standardized medical information and were interviewed after making their decision (which dictated disease management). We evaluated patients' levels of decision-self efficacy (confidence in medical decision-making ability) at the time information was presented and their level of decision satisfaction after finalizing their decision (using standardized questionnaires). We asked patients to explain the reason for their choice and qualitatively analyzed the results.

\footnotetext{
${ }^{1}$ Division of Endocrinology, University Health Network and University of Toronto, Toronto, Canada.

${ }^{2}$ Joint Department of Medical Imaging, University Health Network-Mt Sinai Hospital-Women's College Hospital, University of Toronto, Toronto, Canada.

${ }^{3}$ Department of Otolaryngology-Head and Neck Surgery, University Health Network, Toronto, Canada.

${ }_{5}^{4}$ Department of Surgery, University Health Network and University of Toronto, Toronto, Canada.

${ }^{5}$ Department of Otolaryngology-Head and Neck Surgery/Surgical Oncology, Princess Margaret Cancer Centre, University Health Network and University of Toronto, Toronto, Canada.

${ }^{6}$ Department of Otolaryngology and Head and Neck Surgery, Sunnybrook Health Sciences Centre and University of Toronto, Toronto, Canada.

${ }^{7}$ Department of Otolaryngology and Head and Neck Surgery, Mount Sinai Hospital and University of Toronto, Toronto, Canada.

${ }^{8}$ Department of Otolaryngology and Head and Neck Surgery, Grand River Hospital Corporation, Kitchener, Canada.

${ }^{9}$ Department of Otolaryngology and Head and Neck Surgery, North York General Hospital and University of Toronto, Toronto, Canada.

${ }^{10}$ Division of Endocrinology, Women's College Hospital, Mount Sinai Hospital and University of Toronto, Toronto, Canada.

${ }^{11}$ Department of Otolaryngology and Head and Neck Surgery, William Osler Health System and University of Toronto, Brampton, Canada.

${ }^{12}$ Division of Endocrine Oncology, Princess Margaret Cancer Centre, Toronto, Canada.

${ }^{13}$ Department of Psychosocial Oncology, University Health Network and University of Toronto, Toronto, Canada.

${ }^{14}$ Ted Rogers Centre for Heart Research at Peter Munk Cardiac Centre, Toronto General Hospital Research Institute (TGHRI), University Health Network (UHN), Toronto, Canada.

${ }^{15}$ Institute of Health Policy, Management and Evaluation (IHPME), Dalla Lana School of Public Health, University of Toronto, Toronto, Canada.

${ }^{16}$ Toronto Health Economics and Technology Assessment Collaborative, Toronto General Hospital Research Institute, University Health Network, Toronto, Canada.

${ }^{17}$ Department of Medicine University Health Network and Mount Sinai Hospital and Dalla Lana School of Public Health, University of Toronto, Toronto, Canada.

${ }^{18}$ Department of Health Research Methods, Evaluation and Implementation, Centre for Health Economics and Policy Analysis, McMaster University, Hamilton, Canada.

${ }^{19}$ Department of Surgery, St. Michael's Hospital and University of Toronto, Toronto, Canada.
}

(c) Anna M. Sawka et al., 2020; Published by Mary Ann Liebert, Inc. This Open Access article is distributed under the terms of the Creative Commons Attribution Noncommercial License (http://creativecommons.org/licenses/by-nc/4.0/) which permits any noncommercial use, distribution, and reproduction in any medium, provided the original author(s) and the source are cited. 
Results: We enrolled 74 women and 26 men of mean age 52.4 years, with a mean PTC size of $11.0 \mathrm{~mm}$ (interquartile range $9.0,14.0 \mathrm{~mm})$. Seventy-one patients $(71.0 \%$ [95\% confidence interval 60.9-79.4\%]) chose AS over surgery. Ninety-four percent (94/100) of participants independently made their own disease management choice; the rest shared the decision with their physician. Participants had a high baseline level of decision self-efficacy (mean 94.3, standard deviation 9.6 on a 100-point scale). Almost all (98\%, 98/100) participants reported high decision satisfaction. Factors reported by patients as influencing their decision included the following: perceived risk of thyroidectomy or the cancer, family considerations, treatment timing in the context of life circumstances, and trust in health care providers.

Conclusions: In this Canadian study, $\sim 7$ out of 10 patients with small, low-risk PTC, who were offered the choice of AS or surgery, chose AS. Personal perceptions about cancer or thyroidectomy, contextual factors, family considerations, and trust in health care providers strongly influenced patients' disease management choices.

Keywords: active surveillance, thyroidectomy, medical decision-making, prospective observational study, mixed methods

\section{Introduction}

$\mathbf{T}$ HE INCIDENCE RATE of thyroid cancer, particularly papillary thyroid cancer (PTC), has been increasing throughout the world (1). Globally, thyroid cancer is the 8th most commonly diagnosed malignancy (3rd most common in women); yet, it ranks 25 th in mortality risk (1).

There is substantial regional variation in thyroid cancer incidence. In Canada, some of the highest rates of diagnosis of thyroid cancer are in the province of Ontario (2), particularly the Greater Toronto Area (3). The increase in thyroid cancer incidence in this region has been attributed to the detection of small PTCs, $2 \mathrm{~cm}$ or smaller in maximal diameter (4).

The traditional first step in the management of PTC is surgery (total or hemithyroidectomy), sometimes followed by radioactive iodine (5). Depending on factors such as the extent of thyroidectomy and underlying thyroid function, lifelong thyroid hormone treatment may be required (5). Given some concern about potential overtreatment of small, low-risk PTCs, active surveillance (AS) has been proposed for such tumors (5).

The largest and longest duration prospective study of AS of papillary thyroid microcarcinomas (PTC $\leq 1 \mathrm{~cm}$ in maximal diameter, with no nodal metastases) was initiated in 1993 by Dr. Akira Miyauchi in Kuma Hospital of Japan (6). A second AS study followed in Tokyo in 1995, which included patients with PTC $\leq 2 \mathrm{~cm}$ in maximal diameter, although most of the patients under AS had papillary microcarcinomas (7). To date, there are no reports of any thyroid cancer-related deaths nor any incident distant metastatic disease in PTC patients undergoing AS; furthermore, most patients with PTC under AS have avoided thyroidectomy and thyroid hormone treatment (6-10). Outside of Japan, prospective AS studies for low-risk PTC are in relatively early follow-up stages, with recent reports from ongoing large studies in the United States (11) and Korea (12).

There is little known about AS decision-making in PTC patients. Davies et al. reported that in the Kuma Hospital series, patients under AS did not always receive a choice of AS or surgery (i.e., they sometimes received a single recommendation), although most agreed with the choice of AS at follow-up (13). However, these data may not be generalizable to other cultural contexts or health care settings where patients may want and expect provision of health care information and involvement in medical decision-making.
In May of 2016, we initiated a prospective study offering AS or surgery as options for low-risk PTC $<2 \mathrm{~cm}$ in maximum diameter (14). Our aim was to determine how often low-risk PTC patients offered the options of thyroidectomy or AS, choose AS in lieu of surgery, and to better understand the reasons for their choices. At the time of initiation of this study, we hypothesized that most low-risk PTC patients offered the choice of AS or surgery would choose AS (defined by the lower $95 \%$ confidence interval [CI] of AS selection probability $>50 \%$ ). We herein describe the AS decision-making results of the first 100 consecutive patients enrolled in our study.

\section{Methods}

\section{Study design, population, and recruitment}

We report a mixed-methods analysis on initial real-life medical decision-making outcomes of an ongoing, singlecenter, prospective, observational cohort study in Toronto, Canada. The treatment decision dictated subsequent disease management and was not simply a hypothetical choice. We collected both quantitative and qualitative data (open-ended question) within one survey instrument/questionnaire, which was verbally administered by a research assistant.

Consenting adults (aged $\geq 18$ years) with low-risk PTC $<2 \mathrm{~cm}$ in maximum diameter were eligible in the absence of evidence of the following: (a) concerning encroachment on critical structures (trachea and recurrent laryngeal nerve), (b) radiographic evidence of nodal metastases, (c) radiographic evidence of extrathyroidal extension, and (d) other significant indication(s) for thyroid or parathyroid surgery (14). Additional details about the study have been previously described (Clinicaltrials.gov NCT03271892) (14). Participants were recruited at the University Health Network (UHN) thyroid cancer specialty clinics, which include Otolaryngology, Head and Neck Surgery, Endocrine Surgery, and Endocrinology clinics. $\mathrm{UHN}$ is a regional referral center for thyroid cancer care in the Greater Toronto Area of Ontario, Canada. Referrals are received from primary care physicians as well as regional thyroid cancer specialists. Information about the study was disseminated by the primary investigators (D.P.G. and A.M.S.) to the Toronto area thyroid cancer specialists in lectures and discussions at multiple regional educational events, including rounds, symposia, and journal clubs. The study protocol was also publicly available on Clinicaltrials.gov (NCT03271892) as 
well as a freely accessible online journal protocol article (14). The study was approved by the UHN Research Ethics Board and informed written consent was obtained from all participants. Consecutive eligible patients in participating clinics were considered for study recruitment; eligibility for recruitment was first decided by the consulting surgeon or endocrinologist, followed by an eligibility screen by a research assistant (after signed informed consent for the screen by the patient), and review of the case by a study primary investigator (D.P.G. or A.M.S.) to confirm eligibility. For all study patients, the first available imaging study in our Joint Department of Medical Imaging (which includes UHN, Mount Sinai Hospital, and Women's College Hospital) was reviewed by a study radiologist (S.G.) or primary investigator surgeon experienced in ultrasound (D.P.G.) to ascertain primary tumor location relative to critical structures (trachea and recurrent laryngeal nerve) and the absence of extrathyroidal extension or suspicion of nodal metastases.

All patients consenting to the study participation were provided written information about low-risk PTC prognosis and the options of surgery or AS ("Active Surveillance Option Description"), with the opportunity to review this information in person with a study physician (A.M.S. or D.P.G.). This information included a clear description that surgery was considered the standard of care and that AS was being made available as an option, as part of a research study ("Active Surveillance Option Description" available for review upon request from the corresponding author). Study participants and referring or recruiting physicians were not financially reimbursed for study participation.

\section{Descriptive data collection and outcomes}

Detailed clinical information was collected by a medical record review and review of thyroid cytopathology slides. The patient questionnaires were verbally administered by a research assistant (T.Y.), who noted all of the responses in a paper record and an electronic database. At baseline, patients provided information on demographic characteristics, such as family history, comorbidities (including prior diagnosis of any cancer), and thyroid cancer risks (e.g., family history or history of radiation exposure).

At baseline, patients' level of confidence in making an informed choice on PTC management was evaluated using the Decision Self-Efficacy Scale questionnaire (which includes 11 questions on a 5-point Likert scale, where the final sum is transformed to a score out of 100, with higher levels reflecting higher levels of confidence) $(15,16)$. Participants were interviewed after finalizing their treatment decision and after verbally indicating their treatment choice, as well as their level of decision satisfaction. Decision satisfaction was measured by the Satisfaction with Decision scale (17), which comprised 6 questions, scored on a 5-point Likert scale (overall Satisfaction with Decision score range 5-30, where a score of $\geq 24$ is defined as high decision satisfaction) $(17,18)$.

Patients were asked about the rationale for their choice, specifically "What is the main reason why you decided to have surgery or active surveillance (i.e., no surgery) for your thyroid cancer?" This question referred to the decision for immediate surgery or AS, after provision of information about the choices. Patients' open-ended responses to this question were noted by a research assistant (T.Y.), using patients' own words.

\section{Statistical analysis}

Descriptive analyses were performed using R version 3.4.4. Numerical variables were summarized using mean and standard deviation (SD), or median and interquartile range, as appropriate. Categorical variables were summarized as counts and percentages, with CI for the primary outcome of the treatment choice. Summaries were reported for all patients in the study and for grouping based on surgery or AS. Missing values were omitted from all summaries. In the secondary comparative analyses, the default statistical test was the chisquared test for categorical variables and the unpaired Student's $t$-test for continuous variables. In comparative analyses of categorical variables where any cell count was $<5$, the Fisher's exact test was performed. The Wilcoxon rank-sum test was used in comparing continuous data that were obviously not normally distributed. $p$-Values are reported.

\section{Analysis of qualitative data}

The mixed-methods approach utilized a triangulation design, which combines both quantitative and qualitative research methods, to obtain, analyze, and interpret data (19). The qualitative data (participants' responses to the open-ended question on rationale for the choice of AS or surgery) were independently coded by two investigators (trained in qualitative research), who reviewed the collected data, observed repeated ideas, tagged them with "codes," and extracted them from the data. As more data were analyzed and rereviewed, codes were grouped into concepts and then into categories of concepts, with the aim of explaining participants' explanations for the choice of AS or surgery. This involved a thematic analysis of data, where all final themes were informed by continuous dialogue (discussion and comparison of results) among the coders and a mixed-methods expert investigator (V.E.R.). Comparisons within and across the questionnaire data were conducted (constant comparison technique) (20-23). Multiple readings were used (24) and alternative explanations of the data were explored, to develop the most plausible and robust interpretation of the findings, explaining the rationale for disease management choice $(20,21)$.

The themes were reviewed by two clinicians (A.M.S. and D.G.) to ensure integrity of medical concepts. The final coded data were compared between patient groups choosing surgery or AS. Data were stored and managed electronically using qualitative research software, NVivo 11 (QSR International, Burlington, MA).

\section{Sample size for the interim analysis}

As previously reported (14), we have no a priori calculated sample size for estimating the proportion choosing AS (or surgery); a convenience sample of 100 was used for this initial analysis (which is half of the planned recruitment of 200 patients for the study of longer term outcomes). All 100 patients were asked the qualitative open-ended question, which explored patients' key factors/reasons related to their treatment decision, which secured full thematic saturation in both groups $(19,21,22)$.

\section{Results}

\section{Description of participants}

We screened 132 consecutive patients, of whom 20 were not eligible based on clinical characteristics, 12 declined 


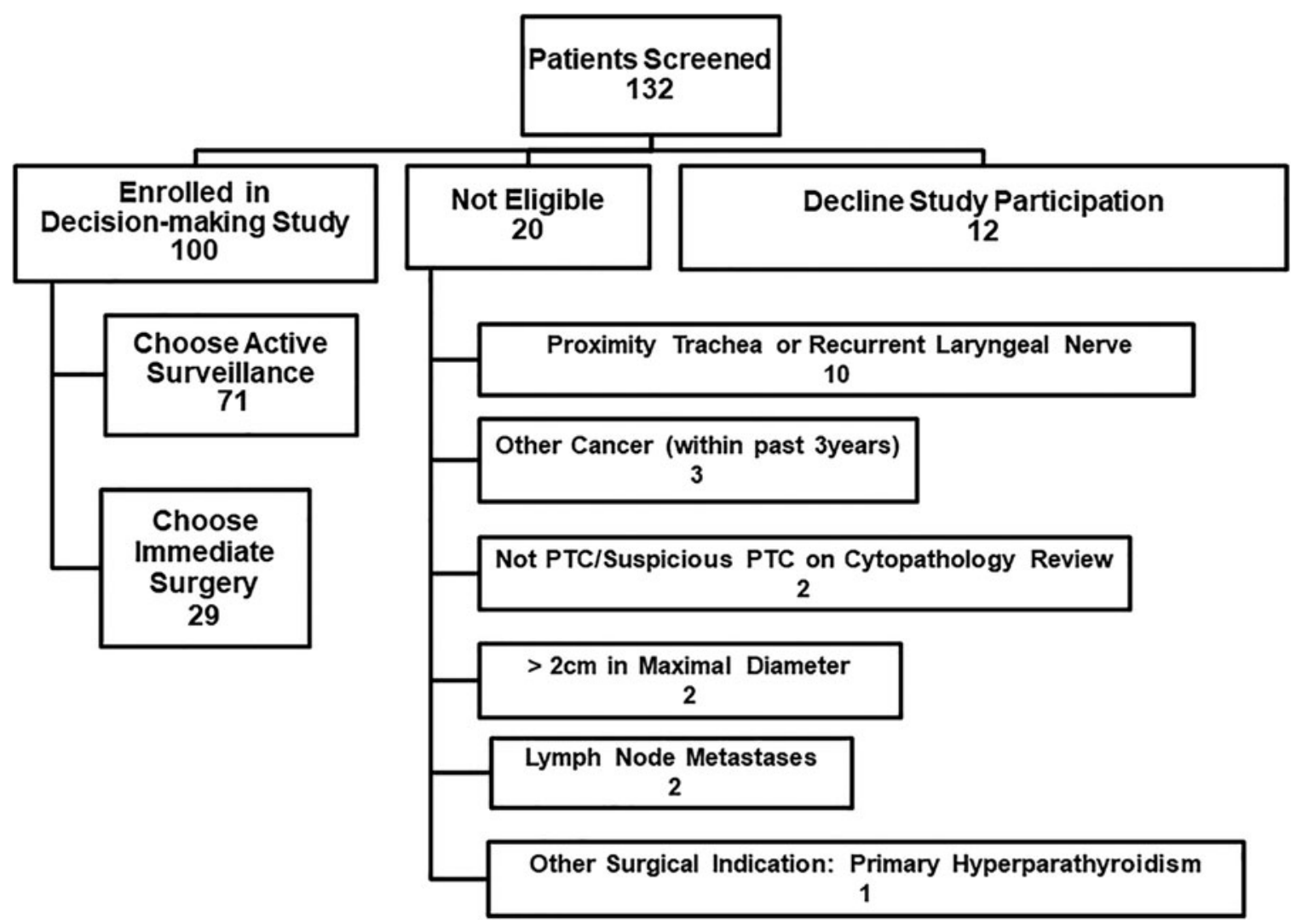

FIG. 1. Participant recruitment flow diagram.

study participation, and 100 enrolled in the study (Fig. 1). The demographic and clinical characteristics of the 100 study participants are shown in Table 1. Approximately threequarters of participants were female, and the mean age was 52.4 years (SD, 15.4), which would be typical of PTC epidemiology. Furthermore, approximately three-quarters of participants were married $(74.0 \%)$ and about three-quarters had children (77/100). Of the 77 individuals reporting having children, slightly under half of individuals reported currently being responsible for raising children younger than 18 years $(34 / 77,44.2 \%)$. About three-quarters of participants (75/100) reported having higher education (college, university, postgraduate, or professional degree). About half of participants were born in Canada (48/99), and $60 \%$ of individuals were white (60/100), with Asian/Southeast Asian (26/100, 26\%) being the next most common race/ethnicity (Table 1). More than half of participants resided in the city of Toronto (52/ $100,52.0 \%$ ).

All participants had a thyroid surgical consultation before enrollment and two-thirds $(68 / 100,68.0 \%)$ identified a UHN thyroid surgeon (head and neck surgeon or endocrine surgeon) their most responsible surgeon, whereas and the rest identified a thyroid surgeon outside of the UHN.

\section{Treatment decisions}

The rate of selection of AS was 71\% (71/100 [CI 61$79 \%$ ]). The mean age of individuals choosing AS (54.8 years, SD 15.6) was 8.5 years higher than that of those choosing surgery (46.3, SD 13.5) $(p=0.012)$. Furthermore, the prev- alence of papillary microcarcinoma was greater in individuals choosing AS (39/71 [54.9\%]) compared with those choosing surgery $(8 / 29[27.6 \%])(p=0.024)$. The sex distribution was similar among those choosing AS (54/71 [76.1\%] female) and choosing surgery (20/29 [69.0\%] female, $p=0.630)$. Among those choosing surgery, 96.6\% (28/29) reported having postsecondary education, whereas $33.8 \%$ (24/71) of those choosing AS had high school as their highest level of education ( $p=0.011$, details in Table 1). Approximately half of all study participants resided in the city of Toronto $(52 / 100,52.0 \%)$, with the proportion of Toronto residents being slightly higher $(56.3 \%)$ in the AS group than in the surgical group $(41.4 \%, p=0.255$, Table 1$)$. However, the proportion of individuals choosing AS was $24 \%$ higher among patients who had a surgeon outside of UHN $(87.5 \%$, $28 / 32$ ), compared with patients who had a surgeon within the institution $(63.2 \%, 43 / 68)(p=0.024)$.

The patients' mean baseline level of decision self-efficacy was high (overall score 94.3, SD 9.6 on a scale where 100 reflects the maximum confidence in the ability to make medical decisions). Decision self-efficacy level did not differ according to education level $(p=0.962)$ nor employment status $(p=0.367)$. Most of the patients $(94 / 100,94 \%)$ indicated that they were the ones who made the final treatment decision, whereas $6 \%(6 / 100)$ indicated they made a shared decision with their physician. The mean score for satisfaction in decision-making was 28.3 out of 30 (SD 2.3); moreover, $98 \%(98 / 100)$ had scores $\geq 24$, indicating high satisfaction with their choice. 
Table 1. Study Participant Demographic and Clinical Characteristics

\begin{tabular}{|c|c|c|c|}
\hline Characteristic & $\begin{array}{c}\text { All patients } \\
(\mathrm{N}=100), \mathrm{n}(\%)\end{array}$ & $\begin{array}{c}\text { Active surveillance } \\
(\mathrm{n}=71[71 \%]), \mathrm{n}(\%)\end{array}$ & $\begin{array}{c}\text { Surgery } \\
(\mathrm{n}=29[29 \%]), \mathrm{n}(\%)\end{array}$ \\
\hline Female sex & $74(74.0)$ & $54(76.1)$ & $20(69.0)$ \\
\hline Mean age (SD) (years) & $52.36(15.41)$ & $54.82(15.55)$ & $46.34(13.52)$ \\
\hline \multicolumn{4}{|l|}{ Marital status } \\
\hline Single & $15(15.0)$ & $11(15.5)$ & $4(13.8)$ \\
\hline Married/common-law & $74(74.0)$ & $50(70.4)$ & $24(82.8)$ \\
\hline Divorced/separated & $5(5.0)$ & $4(5.6)$ & $1(3.4)$ \\
\hline Widowed & $6(6.0)$ & $6(8.5)$ & $0(0)$ \\
\hline Have children & $77(77.0)$ & $55(77.5)$ & $22(75.9)$ \\
\hline \multicolumn{4}{|l|}{ Education } \\
\hline Did not finish high school & $10(10.0)$ & $10(14.1)$ & $0(0)$ \\
\hline Completed high school & $15(15.0)$ & $14(19.7)$ & $1(3.4)$ \\
\hline College or university degree & $50(50.0)$ & $33(46.5)$ & $17(58.6)$ \\
\hline Postgraduate or professional degree & $25(25.0)$ & 14 (19.7) & $11(37.9)$ \\
\hline \multicolumn{4}{|l|}{ Employment } \\
\hline Employed & $67(67.0)$ & $46(64.8)$ & $21(72.4)$ \\
\hline Student (with or without paid work) & $3(3.0)$ & $2(2.8)$ & $1(3.4)$ \\
\hline $\begin{array}{l}\text { Full-time homemaker or caregiver } \\
\text { with no paid employment outside the home }\end{array}$ & $2(2.0)$ & $1(1.4)$ & $1(3.4)$ \\
\hline Unemployed & $6(6.0)$ & $4(5.6)$ & $2(6.9)$ \\
\hline Retired & $22(22.0)$ & $18(25.4)$ & $4(13.8)$ \\
\hline Born in Canada (1 missing response) & $48(48.5)$ & $39(55.7)$ & $9(31.0)$ \\
\hline \multicolumn{4}{|l|}{ 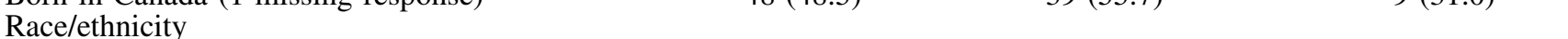 } \\
\hline White & $60(60.0)$ & $39(54.9)$ & $21(72.4)$ \\
\hline Asian/Southeast Asian & $26(26.0)$ & $22(31.0)$ & $4(13.8)$ \\
\hline Middle Eastern & $6(6.0)$ & $3(4.2)$ & $3(10.3)$ \\
\hline Black & $3(3.0)$ & $2(2.8)$ & $1(3.4)$ \\
\hline Other & $5(5.0)$ & $5(7.0)$ & 0 \\
\hline Resident of the city of Toronto at enrollment & $52(52.0)$ & $40(56.3)$ & $12(41.4)$ \\
\hline $\begin{array}{l}\text { Referring surgeon affiliated to } \\
\text { University Health Network }\end{array}$ & $68(68.0)$ & $43(60.6)$ & $25 / 29(86.2)$ \\
\hline $\begin{array}{l}\text { Primary tumor size-median of largest } \\
\text { dimension, mm (interquartile range) }\end{array}$ & $11(8,14)$ & $10(8,13)$ & $14(10,15)$ \\
\hline $\begin{array}{l}\text { Papillary microcarcinoma }(\leq 1 \mathrm{~cm} \text { in } \\
\text { largest dimension) }\end{array}$ & $47(47.0)$ & $39(54.9)$ & $8(27.6)$ \\
\hline Mean thyrotropin concentration (mIU/L) (SD) & $1.76(1.01)$ & $1.61(0.86)$ & $2.15(1.24)$ \\
\hline Currently taking thyroid medication & $11(11.0)$ & $7(9.9)$ & $4(13.8)$ \\
\hline \multicolumn{4}{|l|}{ Thyroid cytologic diagnosis } \\
\hline PTC & $72(72.0)$ & $55(77.5)$ & 17 (58.6) \\
\hline Suspicious for PTC & $28(28.0)$ & $16(22.5)$ & $12(41.4)$ \\
\hline Known family history of thyroid cancer & $15(15.0)$ & $8(11.3)$ & $7(24.1)$ \\
\hline $\begin{array}{l}\text { History of head and neck external } \\
\text { beam radiation treatment }\end{array}$ & $1(1.0)$ & 0 & $1(3.4)$ \\
\hline $\begin{array}{l}\text { History of significant environmental } \\
\text { radiation exposure }\end{array}$ & $3(3.0)$ & $2(2.8)$ & $1(3.4)$ \\
\hline History of workplace radiation exposure & $3(3.0)$ & $2(2.8)$ & $1(3.4)$ \\
\hline History of nonthyroid cancer & $8(8.0)$ & $6(8.5)$ & $2(6.9)$ \\
\hline History of major surgery & $31(31.0)$ & $20(28.2)$ & $11(37.9)$ \\
\hline Current smoking & $4(4.0)$ & $2(2.8)$ & $2(6.9)$ \\
\hline
\end{tabular}

PTC, papillary thyroid cancer; SD, standard deviation.

\section{Qualitative data analysis}

Multiple factors contributed to patients' decisions regarding management of their PTC. In some cases, patients provided complex responses relating to multiple themes. Some themes that were identified across both patient groups and which influenced treatment choices included the following: family history of thyroid or other cancers and previous personal experience with cancer or surgery, family considerations (including the potential impact of PTC or its treatment on the family and the opinions of family members), timing of surgical treatment relative to life circumstances, and trust in health care providers and their care plans (details with representative patient reflections in Fig. 2). Some variations of themes were group-specific, specifically: (a) AS group-particular concern about the risks of surgery, the possibility of having to take thyroid hormone treatment, and the potential impact on quality of life, and (b) surgical 
A

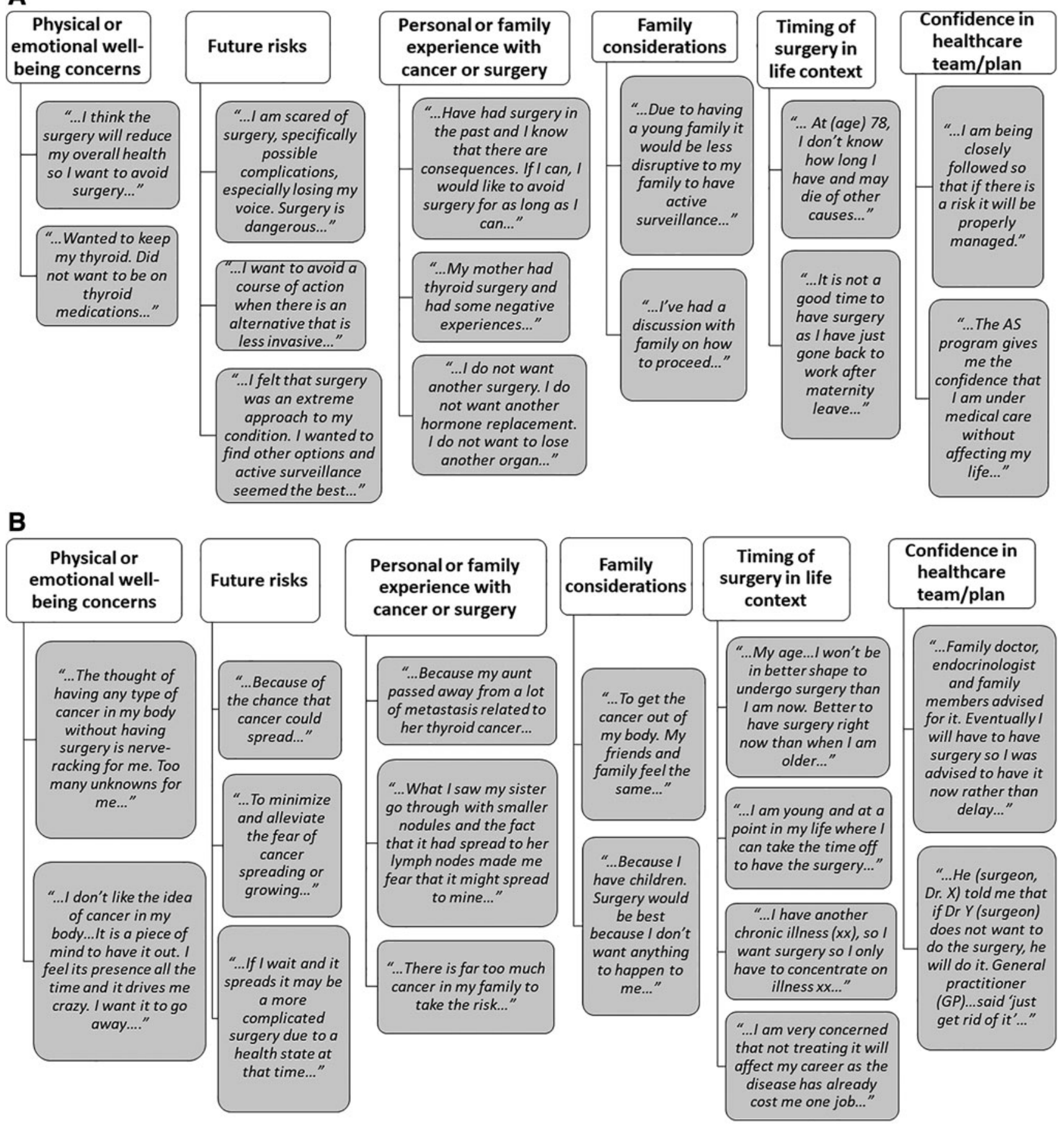

FIG. 2. (A) Themes and reflections from patients choosing active surveillance. (B) Themes and reflections from patients choosing surgery.

group-particular concern about emotional well-being, particularly the anxiety/emotional stress associated with having a cancer diagnosis and not curing it with surgery. In addition, for some individuals in the surgical group, having an option for a hemithyroidectomy, as opposed to a total thyroidectomy, was considered less aggressive and not as threatening. For example, one individual in the surgical group indicated "...Because it is only a hemi, the long-term consequences are not as severe as a total thyroidectomy..." and another indicated, "... Was told I will only need to have half my thyroid removed therefore I will not need thyroid replacement medication after the surgery..."

Additional comments from patients in both the AS and surgical groups reflected that they placed value on the medical information provided in the study as well as the opportunity to participate in disease management decision-making. 
For example, one of the AS group participants indicated "...The study is helping me be better informed about my disease...," another indicated, " ...the information helped me make my decision...," and a third indicated, "Dr X relayed the Japanese study results to me and having a choice was important. I wanted to let people in the future to have a choice..." Furthermore, two patients who chose surgery remarked, "...I had a lot of time to think about my choice and better education about my choice..." and "I have considered all options and professional opinions and I have decided that surgery is best for me..."

\section{Discussion}

In this prospective observational study, we found that $71 \%$ of patients with low-risk PTC chose to undergo AS, if given this real-life choice as an alternative to immediate surgery. The level of decision self-efficacy of patients in this study was high (reflecting a high level of confidence in decision-making ability); as such, most patients made their own treatment decision. Moreover, the vast majority of patients were highly satisfied with their decision, which dictated subsequent disease management. Patients from both the surgery and AS groups indicated that they valued provision of medical information and being involved in their treatment choice. Furthermore, only 9\% (12/132) of potentially eligible patients declined study participation, suggesting strong patient interest in a thyroid cancer treatment medical decision-making study.

In this study, the mean age of individuals in the AS group was higher than that in the surgical group; furthermore, the prevalence of papillary microcarcinoma was higher in the AS group compared with the surgical group. These findings may reflect some of the currently known evidence on this topic. Ito et al. have reported that older patients may experience lower rates of disease progression under AS, compared with younger individuals (10). As such, AS may be a more attractive option for some older individuals and their physicians. Furthermore, most of the literature on AS is focused on papillary microcarcinoma, with only a couple of recently published studies reporting on outcomes of a relatively small number of individuals with PTC larger than $1 \mathrm{~cm}(7,11)$. It is possible that both patients and their physicians may be less comfortable with AS for very young patients who would require prolonged follow-up or in those with PTC $>1 \mathrm{~cm}$ in size (given a paucity of evidence reported on long-term outcomes in patients with larger tumors).

Patients in our study reported multiple factors that influenced the final decision on AS or surgery, including the perceived threat/risk of the disease or its treatment, consideration of long-term physical and emotional well-being (including the potential use of thyroid hormone), family, life circumstances, trust in health care providers, and for individuals in the surgery group, the type of surgery being recommended (i.e., hemithyroidectomy as opposed to total thyroidectomy).

This is the first prospective study in which a qualitative analysis has explored patients' rationale for AS or surgery at the time the real-life decision is finalized. Our findings generally confirm the findings of D'Agostino et al. who reported on 15 Americans with papillary microcarcinoma (and six caregivers) who had been offered the options of AS or surgery in the past (25). In the American study, individuals who chose AS viewed papillary microcarcinoma as "a common, indolent, and low-risk disease," expressing concerns about life after thyroidectomy and thyroid hormone treatment, and they appreciated the flexibility in timing of surgery (24). As reflected in our study, D'Agostino et al. stressed a "deep level of trust" in health care practitioners and their institutions (25). Davies et al. recently reported that patients with papillary microcarcinoma, who were under AS at the Kuma Hospital, worried about disease progression or the possibility of needing surgery (13), however, not all of these patients were given a choice in disease management, which may potentially contribute to the findings.

Our study was prospective, utilizing a standardized approach for provision of medical information to patients, with a mixed-methods approach using a large qualitative sample to further validate and elaborate on our quantitative data. An important strength is that the information collected was in the context of a real-life treatment decision, as opposed to a hypothetical choice.

However, there are limitations of this study. First, the sample size for quantitative outcomes is not large. Since we are continuing study recruitment and further follow-up, we as of yet have insufficient data on long-term outcomes. Furthermore, our study is subject to potential referral bias, which may have been reflected by a relatively higher proportion of patients with a surgeon outside UHN selecting AS, compared with those with a UHN surgeon. Furthermore, given that information about our study was publicly available and registered at Clinicaltrials.gov, patients were free to contact our study staff directly to inquire about the study or ask their doctors for a referral to one of the study physicians. These factors may have contributed to an increased rate of selection of AS, in individuals who were already interested in AS. Individuals who already had learned about AS in advance of the referral (from their own research of the literature or based on discussions with the referring doctor) may have already been more confident in their preference in AS at the time of evaluation, potentially impacting decision self-efficacy and related decisionmaking variables. Furthermore, we did not have audio- or video-recordings of the interactions of patients with referring nor study physicians, so detailed analyses of such interactions could not be performed. Another limitation is that other than education level and employment status, we did not collect detailed information on socioeconomic status, such as personal or family income. However, as the study was conducted in a publicly funded health care system, it is unlikely that socioeconomic status presented a major barrier to health care access. Our qualitative analysis is also limited by collection of data relating to one open-ended question on rationale for treatment choice; in-depth analysis of other concepts was not performed.

In conclusion, this study found that a majority of patients with small, low-risk PTC chose AS, if offered this option as an alternative to thyroidectomy. Our patients were generally confident in medical decision-making, and at least in the short-term, largely satisfied with their choice. Furthermore, our patients valued receiving medical information and participation in decision-making. Personal perceptions about 
cancer or thyroidectomy, contextual factors, family considerations, and trust in health care providers strongly influenced treatment choices. Continued research is needed to determine the long-term clinical and psychosocial outcomes of AS and surgery in this population.

\section{Acknowledgments}

The authors thank Mrs. Coreen Marino and Ms. Sindy Prasad for assistance in arranging patients' clinical and diagnostic testing appointments for the study. The authors also thank Mr. John Matelski who assisted in the statistical analyses and Troy Francis who assisted with the data coding.

\section{Author Disclosure Statement}

No competing financial interests exist.

\section{Funding Information}

The authors are currently involved in ongoing research in AS of low-risk PTC, funded by the Canadian Institutes of Health Research and the Canadian Cancer Society Research Institute. This study was funded by the following operating grants: Ontario Academic Health Sciences Centres Alternate Funding Plan Innovation Grant (Ontario Ministry of Health); Canadian Cancer Society Research Institute (Lotte and John Hecht Memorial Foundation Innovation Grant, no. 703948). There are no other relevant financial disclosures.

\section{References}

1. Wiltshire JJ, Drake TM, Uttley L, Balasubramanian SP 2016 Systematic review of trends in the incidence rates of thyroid cancer. Thyroid 26:1541-1552.

2. Topstad D, Dickinson JA 2017 Thyroid cancer incidence in Canada: a national cancer registry analysis. CMAJ Open 5: E612-E616.

3. Corsten MJ, Hearn M, McDonald JT, Johnson-Obaseki S 2015 Incidence of differentiated thyroid cancer in Canada by City of residence. J Otolaryngol Head Neck Surg 44:36.

4. Kent WD, Hall SF, Isotalo PA, Houlden RL, George RL, Groome PA 2007 Increased incidence of differentiated thyroid carcinoma and detection of subclinical disease. CMAJ 177:1357-1361.

5. Haugen BR, Alexander EK, Bible KC, Doherty GM, Mandel SJ, Nikiforov YE, Pacini F, Randolph GW, Sawka AM, Schlumberger M, Schuff KG, Sherman SI, Sosa JA, Steward DL, Tuttle RM, Wartofsky L 20162015 American Thyroid Association management guidelines for adult patients with thyroid nodules and differentiated thyroid cancer: the American Thyroid Association Guidelines Task Force on Thyroid Nodules and Differentiated Thyroid Cancer. Thyroid 26:1-133.

6. Ito Y, Miyauchi A, Kudo T, Oda H, Yamamoto M, Sasai H, Masuoka H, Fukushima M, Higashiyama T, Kihara M, Miya A 2018 Trends in the implementation of active surveillance for low-risk papillary thyroid microcarcinomas at Kuma Hospital: gradual increase and heterogeneity in the acceptance of this new management option. Thyroid 28: 488-495.

7. Sakai T, Sugitani I, Ebina A, Fukuoka O, Toda K, Mitani H, Yamada K 2019 Active surveillance for T1bN0M0 papillary thyroid carcinoma. Thyroid 29:59-63.
8. Oda H, Miyauchi A, Ito Y, Yoshioka K, Nakayama A, Sasai H, Masuoka H, Yabuta T, Fukushima M, Higashiyama T, Kihara M, Kobayashi K, Miya A 2016 Incidences of unfavorable events in the management of low-risk papillary microcarcinoma of the thyroid by active surveillance versus immediate surgery. Thyroid 26:150155.

9. Miyauchi A, Kudo T, Ito Y, Oda H, Sasai H, Higashiyama T, Fukushima M, Masuoka H, Kihara M, Miya A 2018 Estimation of the lifetime probability of disease progression of papillary microcarcinoma of the thyroid during active surveillance. Surgery 163:48-52.

10. Ito Y, Miyauchi A, Kihara M, Higashiyama T, Kobayashi $\mathrm{K}$, Miya A 2014 Patient age is significantly related to the progression of papillary microcarcinoma of the thyroid under observation. Thyroid 24:27-34.

11. Tuttle RM, Fagin JA, Minkowitz G, Wong RJ, Roman B, Patel S, Untch B, Ganly I, Shaha AR, Shah JP, Pace M, Li D, Bach A, Lin O, Whiting A, Ghossein R, Landa I, Sabra M, Boucai L, Fish S, Morris LGT 2017 Natural history and tumor volume kinetics of papillary thyroid cancers during active surveillance. JAMA Otolaryngol Head Neck Surg 143:1015-1020.

12. Kong SH, Ryu J, Kim MJ, Cho SW, Song YS, Yi KH, Park DJ, Hwangbo Y, Lee YJ, Lee KE, Kim SJ, Jeong WJ, Chung EJ, Hah JH, Choi JY, Ryu CH, Jung YS, Moon JH, Lee EK, Park YJ 2019 Longitudinal assessment of quality of life according to treatment options in low risk papillary thyroid microcarcinoma patients-active surveillance or immediate surgery (interim analysis of MAeSTro). Thyroid 29:1089-1096.

13. Davies L, Roman BR, Fukushima M, Ito Y, Miyauchi A 2019 Patient experience of thyroid cancer active surveillance in Japan. JAMA Otolaryngol Head Neck Surg 145: 363-370.

14. Sawka AM, Ghai S, Tomlinson G, Rotstein L, Gilbert R, Gullane P, Pasternak J, Brown D, de Almeida J, Irish J, Chepeha D, Higgins K, Monteiro E, Jones JM, Gafni A, Goldstein DP 2018 A protocol for a Canadian prospective observational study of decision-making on active surveillance or surgery for low-risk papillary thyroid cancer. BMJ Open 8:e020298.

15. O'Connor AM 2002 User Manual-Decision Self-Efficacy Scale. Ottawa Hospital Research Institute, Ottawa. Available at: https://decisionaid.ohri.ca/docs/develop/User_ Manuals/UM_Decision_SelfEfficacy.pdf (accessed June 26, 2019).

16. Bunn H, O'Connor A 1996 Validation of client decisionmaking instruments in the context of psychiatry. Can J Nurs Res 28:13-27.

17. Holmes-Rovner M, Kroll J, Schmitt N, Rovner DR, Breer ML, Rothert ML, Padonu G, Talarczyk G 1996 Patient satisfaction with health care decisions: the satisfaction with decision scale. Med Decis Making 16:58-64.

18. Hitz F, Ribi K, Li Q, Klingbiel D, Cerny T, Koeberle D 2013 Predictors of satisfaction with treatment decision, decision-making preferences, and main treatment goals in patients with advanced cancer. Support Care Cancer 21: 3085-3093.

19. Creswell JW, Piano Clark VL 2007 Designing and Conducting Mixed Methods Research. Sage Publications, Los Angeles, CA.

20. Bryman A 1988 Quantity and Quality in Social Research. Routledge, London, UK. 
21. Eakin JM, Mykhalovskiy E 2003 Reframing the evaluation of qualitative health research: reflections on a review of appraisal guidelines in the health sciences. J Eval Clin Pract 9:187-194.

22. Corbin JM, Strauss AL 2008 Basics of Qualitative Research: Techniques and Procedures for Developing Grounded Theory. Third edition. Sage Publications, Los Angeles, CA.

23. Kvale S 1996 Interviews: An Introduction to Qualitative Research Interviewing. Sage Publications, Thousand Oaks, CA.

24. Sturges JE, Hanrahan KJ 2004 Comparing telephone and face-to-face qualitative interviewing: a research note. Qual Res 4:107-118.
25. D'Agostino TA, Shuk E, Maloney EK, Zeuren R, Tuttle RM, Bylund CL 2018 Treatment decision making in early-stage papillary thyroid cancer. Psychooncology 27: 61-68.

Address correspondence to: Anna M. Sawka, MD, PhD, FRCPC University Health Network - Toronto General Hospital 200 Elizabeth Street Toronto 12 EN-212

Ontario

Canada

E-mail: sawkaam@yahoo.com 